\title{
Overexpression of microRNA-133b is associated with the increased survival of patients with hepatocellular carcinoma after curative hepatectomy: Involvement of the EGFR/PI3K/Akt/mTOR signaling pathway
}

\author{
XUEDONG WANG $^{1,2}$, JIANPING ZENG $^{1,2^{*}}$, LIANG WANG $^{1 *}$, XINJING ZHANG $^{3}$, \\ ZHIQIAN LIU ${ }^{2}$, HONGYI ZHANG ${ }^{1,3}$ and JIAHONG DONG ${ }^{1,2,3}$ \\ ${ }^{1}$ Department of Hepatobiliary Surgery, Beijing Tsinghua Changgung Hospital, Medical Center, \\ Tsinghua University, Beijing 102218; ${ }^{2}$ Department of Hepatobiliary Surgery, Chinese General PLA Hospital, \\ Beijing $100853 ;{ }^{3}$ Medical School of Tsinghua University, Beijing 100084, P.R. China
}

Received June 25, 2016; Accepted August 9, 2016

DOI: 10.3892/or.2017.5699

\begin{abstract}
The aim of the present study was carried out to investigate the association of microRNA-133b (miRNA-133b) expression with the prognosis of patients with hepatocellular carcinoma (HCC) after curative hepatectomy. In the present study, the expression of miRNA-133b in HCC tissues was determined to be lower than that noted in the adjacent normal tissues. Overall and disease-free survival of the HCC patients with high miRNA-133b expression was observably extended, compared with the HCC patients with low miRNA-133b expression. Moreover, the overexpression of miRNA-133b inhibited cell proliferation, increased lactate dehydrogenase (LDH) activity, induced apoptosis and promoted caspase-3/-8 activities and $\mathrm{Bax} / \mathrm{Bcl}-2$ protein expression in $\mathrm{HCC}$ cells, whereas the protein expression of epidermal growth factor receptor (EGFR) was significantly decreased. The overexpression of miRNA-133b significantly suppressed PI3K, phosphorylated (p)-Akt and p-mTOR protein expression in HCC cells. GW2974, an EGFR inhibitor, suppressed the protein expression of EGFR, inhibited cell proliferation, increased LDH activity, induced apoptosis and promoted caspase-3/-8 activities and Bax/Bcl-2 protein expression, downregulated PI3K, p-Akt and p-mTOR protein expression in the transfected HCC cells overexpressing miRNA-133b. Taken together, our results
\end{abstract}

Correspondence to: Dr Xuedong Wang, Department of Hepatobiliary Surgery, Beijing Tsinghua Changgung Hospital, Medical Center, Tsinghua University, 168 Litang Road, Changping, Beijing 102218, P.R. China

E-mail: wangxuedong301@163.com

${ }^{*}$ Contributed equally

Key words: microRNA-133b, hepatocellular carcinoma, EGFR, PI3K-Akt, mTOR indicate that the overexpression of miRNA-133b is associated with the increased survival of HCC patients after curative hepatectomy. The effects of miRNA-133b in HCC are mediated through the EGFR/PI3K/Akt/mTOR signaling pathway.

\section{Introduction}

Hepatocellular carcinoma (HCC) is one of the most common malignant tumors (1). According to data since 2010, HCC ranks fifth among the most commonly diagnosed malignant tumors in men and eighth in women (1). The number of patients diagnosed with HCC has continued to increase (2). The disease can be radically cured by surgical removal; the first choice for HCC treatment in China and many other countries (3). Through ultrasound screening for patients with liver cirrhosis or hepatitis, numerous patients suitable for hepatectomy have been identified (4). Moreover, with the development of surgical technology and preoperative management, hepatectomy for $\mathrm{HCC}$ has become a safe surgery with rare complications (4). Over the past 30 years, the survival rate of HCC patients who have undergone hepatectomy has been markedly improved (5). Nevertheless, HCC mainly follows the occurrence of viral hepatitis and liver cirrhosis in China and Southeast Asia (6). The long-term effects are unsatisfactory. Postoperative occurrence is the primary cause of treatment failure (7).

Neoplasm staging is of great significance (5). It is conducive to the development of treatment strategies and the prognostic assessment of patients (8). However, it provides standardized platforms for the evaluation of new treatment methods and the comparison of curative effects. As for HCC, there are many treatments to this disease, including surgical re-resection, TACE, PEIT, RFA, microwave therapy, radiotherapy, cryotherapy and liver transplant (9). Wherein, surgical re-resection is still the most effective treatment (9). The appropriate treatment should be selected according to the clinicopathological features of the recurrent carcinoma, characteristics of the patients, reserve functions of the liver and the general conditions of the patients (7). 
Recurrence and metastasis are important factors influencing the curative effects. Since the time of intrahepatic recurrence affects the survival rate of patients after recurrence, there is an urgent need for indicators able to accurately predict the postoperative prognosis of HCC patients (10). Currently, there are numerous studies concerning the prognostic factors of HCC after surgical re-resection both at home and abroad (11). These factors include clinicopathological factors of the primary and recurrent tumors, the origin of HCC, time of recurrence, general conditions of the patients and surgical methods (11). However, due to the different liver diseases and non-uniform standard for radical resection, there are differences in the markers influencing the postoperative prognosis of HCC patients (12).

The mature microRNA (miRNA) is a type of non-coding and single-stranded RNA molecule with 19-25 nucleotides (10). The precursor of miRNA is referred to as pri-miRNA. It is double-stranded RNA consisting of 70-100 nucleotides. After cutting and unwinding, it develops into mature miRNA (13). miRNA sequences are highly conserved among different species, which indicates that these molecules play an important role in the development, proliferation, differentiation and apoptosis in organisms (13). However, a number of studies suggest that there are obvious differences in the miRNA profile in different diseases and on gene expression $(13,14)$. This indicates that certain miRNAs can be regarded as biomarkers and used as diagnostic and prognostic indicators of malignant tumors (15).

A large amount of research results have shown that the overexpression or mutation of epidermal growth factor receptor (EGFR) exists in many tumor cells and tumor tissues (16). EGFR refers to the transmembrane receptor with RTK activity. It is coded by c-erbB1 genes. The ligands of EGFR include EGF, TGF, AREG, HB-EGF, EPR and $\beta$-cytokine (17). It regulates the biological activity of cells by mediating various signaling pathways (18). The overexpression or mutation of EGFR is often associated with the poor prognosis, rapid metastasis, short-term recurrence and short survival time of epithelium tumors such as breast, gastrointestinal, ovarian and cervical cancer (19).

PI3K is a kinase that is involved in multiple cellular signaling pathways. As regards Akt, it is an important factor in the downstream of PI3K (20). It is able to regulate cell proliferation and prevent cell apoptosis. PI3K/Akt is one of the principal downstream signaling pathways of the ERBB family of receptor tyrosine kinases (21). The cytoplasmic domain of ERBB3 activates PI3K through the phosphorylation of tyrosine. The activation of a catalytic subunit of PI3K leads to the excessive activation of PI3K in a variety of tumor tissues (22).

Akt is a serine/threonine-specific protein kinase. Since it is highly homologous with PKA and PKC, it is also referred to as protein kinase $\mathrm{B}$ (PKB) (23). It plays an important role in the regulation of cell growth, proliferation and survival and glucose metabolism. AKT is able to inhibit Smac/DIABLO, activate and upregulate apoptosis-inducing factors and inhibit p53 with Mdm2 (23). In addition, it enables the inhibitory factors of CDK (p2 $1^{\mathrm{CIP} 1 / \mathrm{WAF} 1}$ and $\mathrm{p} 27^{\mathrm{KIP} 1}$ ) to move out of the cell nucleus and degrade in the cytoplasm through phosphorylation, thereby promoting cell proliferation.

mTOR is an important site in the downstream of the PI3K signaling pathway. It plays a critical role in tumor develop- ment, invasion, metastasis and angiogenesis. In $90-100 \%$ of HCC cases, the AKT/mTOR signaling pathway is activated, which is significantly correlated with the recurrence of tumors and reduced survival rate of patients (21). In this study, we evaluated the possible associations between microRNA-133b (miRNA-133b) and the prognosis of patients with HCC in light of clinicopathological characteristics after curative hepatectomy, and investigated the effect of miRNA-133b on the EGFR/PI3K/Akt/mTOR signaling pathway.

\section{Materials and methods}

Patients and follow-up. We analyzed the prospective collected data of 112 HCC patients after curative hepatectomy between February 2010 and May 2011 at the Chinese General PLA Hospital. The present study was approved by the Institutional Ethics Committee of the Chinese General PLA Hospital. Written informed consent was obtained from all patients. Basic clincal characteristics of all patient with HCC were collected and are documented in Table I. During the first 2 years after surgery, patients were followed-up every 2 months, and from 3 years after surgery, patients were followed-up every 3-4 months.

Reverse transcriptase-quantitative polymerase chain reaction $(R T-q P C R)$. The miRNA-133b expression levels in HCC tissues and matched adjacent normal tissues were detected using qRT-PCR. Total RNA was extracted from HCC tissue samples and adjacent normal tissues using TRIzol (Invitrogen, Grand Island, NY, USA) according to the manufacturer's instructions. Specific cDNA was synthesized from total RNA (5-10 ng) using TaqMan MicroRNA assays protocol (Applied Biosystems, Foster City, CA, USA). qRT-PCR (7900HT Fast Real-Time PCR System) was performed using GeneAmp ${ }^{\circledR}$ Fast PCR Master Mix (both from Applied Biosystems, Thermo Fisher Scientific, Inc., Waltham, MA, USA) to analyze the expression level of miRNA-133b. The qPCR conditions were as follows: $95^{\circ} \mathrm{C}$ for $5 \mathrm{~min} ; 40$ cycles of $94^{\circ} \mathrm{C}$ for $30 \mathrm{sec} ; 60^{\circ} \mathrm{C}$ for $45 \mathrm{sec} ; 72$ for $45 \mathrm{sec}$. The following primers were used: miR-133b forward, 5'-GAACCAAGCCGCCCGAGA-3' and reverse, 5'-CCGCCCTGCTGTGCTGGT-3'; RNU6B was used as internal control: U6 forward, 5'-CTCGCTTCGGCAGC ACA-3' and reverse, 5'-AACGCTTCACGAATTTGCGT-3'. Relative quantification of miRNA-133b expression was evaluated using the formula, $2^{-\Delta \Delta \mathrm{Ct}}$.

Cell culture. Human HCC cell lines HepG2, SMMC7721, Bel7404 and HCCM3 were purchased from the Shanghai Cell Bank of the Chinese Academy of Sciences (Shanghai, China) and cultured in Dulbecco's modified Eagle's medium (DMEM), supplemented with $10 \%$ fetal bovine serum (FBS) (both from Invitrogen; Thermo Fisher Scientific, Inc.) $100 \mathrm{U} /$ $\mathrm{ml}$ penicillin and $100 \mu \mathrm{g} / \mathrm{ml}$ streptomycin in a humidified atmosphere of $5 \% \mathrm{CO}_{2}$ at $37^{\circ} \mathrm{C}$.

miRNA transfection. The HepG2 cells $\left(1 \times 10^{5}\right.$ cells/well) were seeded into 6-well plates and cultured overnight. Then, the cells were transfected with $100 \mathrm{nM}$ of negative control mRNA or the miR-133b mimics (both from Shanghai GenePharma Co., Ltd., Shanghai, China) using Lipofectamine $2000^{\circledR}$ (Invitrogen; Thermo Fisher Scientific, Inc.). At 48 h post-transfection, the 
Table I. Clinical characteristics of the patients with HCC.

\begin{tabular}{lcc}
\hline Variables & $\begin{array}{c}\text { All patients } \\
(\mathrm{n}=112)\end{array}$ & P-value \\
\hline Age (years) & & 0.731 \\
$\quad \leq 55$ & 63 & \\
$>55$ & & 0.942 \\
Sex, $\mathrm{n}$ & 58 & \\
Female & 54 & 0.178 \\
Male & & \\
Tumor size (cm), $\mathrm{n}$ & 49 & 0.063 \\
$\leq 3.0$ & 63 & \\
$>3.0$ & & \\
Edmondson grade, $\mathrm{n}$ & 12 & \\
I & 67 & \\
II & 33 & \\
II & $75.2 \pm 216.8$ & \\
Serum AFP levels $(\mathrm{ng} / \mathrm{ml})$ & $38.2 \pm 4.5$ & \\
Albumin $(\mathrm{g} / \mathrm{l})$ & $19.4 \pm 11.2$ & \\
Bilirubin $(\mu$ mol/l) & & \\
\hline
\end{tabular}

HCC, hepatocellular carcinoma.

transfected HepG2 cells were prepared for further analysis. EGFR inhibitor (GW2974 $2 \mu \mathrm{M}$ Sigma Chemical Co. St. Louis, MO, USA) was added into cell after transfection at $4 \mathrm{~h}$.

Cell viability assay. The transfected HepG2 cells $\left(1 \times 10^{4}\right.$ cells/well) were seeded into 96-well plates and cultured overnight. Cell viability was cultured using the 3-(4,5-dimethylthiazol-2-yl)-2,5-diphenyltetrazolium bromide (MTT; Invitrogen; Thermo Fisher Scientific, Inc.) assay for $4 \mathrm{~h}$ in a humidified atmosphere of $5 \% \mathrm{CO}_{2}$ at $37^{\circ} \mathrm{C}$. The medium was replaced, and then dimethyl sulfoxide (DMSO) (150 $\mu \mathrm{l})$ was added into every well and shaken for $20 \mathrm{~min}$ at $37^{\circ} \mathrm{C}$. Absorbance was measured using the EL800 Universal Microplate Reader (BioTek Instruments, Inc., Winooski, VT, USA) at $570 \mathrm{~nm}$.

Measurements of lactate dehydrogenase ( $\mathrm{LDH}$ ) activity. The transfected HepG 2 cells $\left(1 \times 10^{4}\right.$ cells/well) were seeded into 96-well plates and cultured overnight. Cell viability was cultured using lactate dehydrogenase for $1 \mathrm{~h}$ at $37^{\circ} \mathrm{C}$ in darkness. Absorbance was measured using the EL800 Universal Microplate Reader at $490 \mathrm{~nm}$.

Measurements of the apoptosis rate. The transfected HepG2 cells $\left(1 \times 10^{5}\right.$ cells/well $)$ were seeded into 6 -well plates and cultured overnight. The HepG2 cells were added together with $195 \mu \mathrm{l}$ Annexin V-FITC binding buffer and $5 \mu \mathrm{l}$ Annexin V-FITC, and incubated at room temperature in the dark for $10 \mathrm{~min}$. Then, the cells were stained with $10 \mu \mathrm{l}$ propidium iodide (PI) at room temperature in the dark for $10 \mathrm{~min}$. The apoptosis ratio was recognized with the flow cytometer FACSVerse (Becton-Dickinson, Heidelberg, Germany).

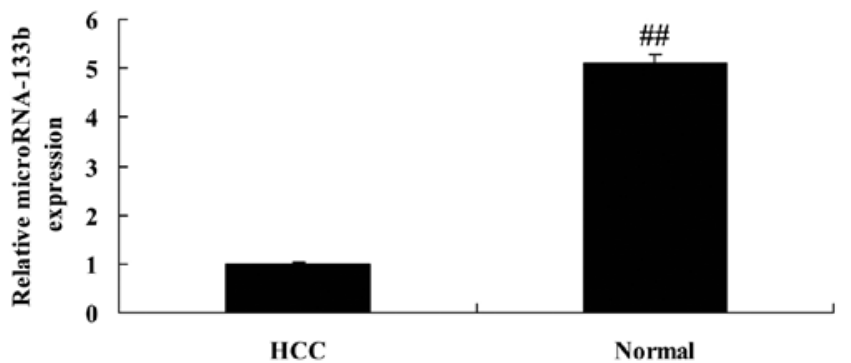

Figure 1. microRNA-133b expression in patients with HCC after curative hepatectomy. HCC, hepatocellular carcinoma; normal, adjacent normal tissues; ${ }^{\# \#} \mathrm{p}<0.01$ compared with the HCC group.

Measurements of caspase-3/-8 activity. The transfected HepG 2 cells $\left(1 \times 10^{5}\right.$ cells/well) were seeded in 6 -well plates and cultured overnight. The HepG2 cells were incubated with Ac-DEVD- $p$ NA (caspase-3 activity) and Ac-IETD- $p$ NA (caspase- 8 activity) at room temperature in the dark for $2 \mathrm{~h}$. Absorbance was measured using the EL800 Universal Microplate Reader at $405 \mathrm{~nm}$.

Western blot analysis. The transfected HepG2 cells $\left(1 \times 10^{5}\right.$ cells/well) were seeded in 6-well plates and cultured overnight. Cells were washed with ice-cold phosphate-buffered saline (PBS) and obtained by resuspending the cells in RIPA buffer kit (Beyotime Institute of Biotechnology, Shanghai, China). The protein concentration was determined using the Bradford protein assay kit (Beyotime Institute of Biotechnology). Protein $(50 \mu \mathrm{g})$ was subsequently separated using $10-12 \%$ sodium dodecyl sulfate-polyacrylamide gel electrophoresis and electrotransferred onto nitrocellulose membranes (EMD; Millipore, Billerica, MA, USA). The membranes were blocked using 5\% skim milk powder and incubated overnight with primary antibodies: anti-Bax, anti-Bcl-2, anti-PI3K, antip-Akt, anti-p-mTOR and GAPDH at $4^{\circ} \mathrm{C}$ overnight. The membranes were then washed with Tris-buffered saline with Tween-20 (TBST) and incubated with HRP-conjugated goat anti-rabbit immunoglobulin $\mathrm{G}$ for $1 \mathrm{~h}$ at room temperature. Protein bands were visualized with the Chemi-Lumi One L western blotting substrate.

Statistical analysis. All data are presented as the mean \pm standard error. The Kaplan-Meier method was used to estimate survival rates, and the log-rank test was used to assess survival differences between groups. Data from each group were statistically analyzed using the Student's t-test. Differences were considered statistically significant at a $\mathrm{p}$-value of $<0.05$.

\section{Results}

Expression of miRNA-133b in patients with HCC after curative hepatectomy. We firstly assayed the expression of miRNA-133b in patients with HCC after curative hepatectomy. As shown in Fig. 1, the expression of miRNA-133b in the HCC tissues was effectively lower than that in the adjacent normal tissues. We then assessed that the correlation between miRNA-133b expression and clinicopathological features of $\mathrm{HCC}$ and found that the tumor size of the HCC patients with low miRNA-133b expression was larger than that of the HCC 
Table II. Correlation between microRNA-133b expression and clinicopathological features of HCC.

\begin{tabular}{|c|c|c|c|c|}
\hline Variables & All patients $(n=112)$ & Low miRNA-133b & High miRNA-133b & P-value \\
\hline Age (years) & & & & 0.892 \\
\hline$\leq 55$ & 63 & 35 & 28 & \\
\hline$>55$ & 49 & 23 & 26 & \\
\hline Sex & & & & 0.933 \\
\hline Female & 58 & 31 & 27 & \\
\hline Male & 54 & 28 & 26 & \\
\hline Tumor size $(\mathrm{cm})$ & & & & 0.009 \\
\hline$\leq 3.0$ & 49 & 35 & 14 & \\
\hline$>3.0$ & 63 & 48 & 15 & \\
\hline Edmondson grade & & & & 0.011 \\
\hline $\mathrm{I}$ & 12 & 7 & 5 & \\
\hline II & 67 & 48 & 19 & \\
\hline III & 33 & 25 & 8 & \\
\hline Serum AFP levels (ng/ml) & $75.2 \pm 216.8$ & $108.2 \pm 301.2$ & $53.7 \pm 167.2$ & 0.001 \\
\hline Albumin (g/l) & $38.2 \pm 4.5$ & $39.9 \pm 6.1$ & $37.3 \pm 5.9$ & 0.371 \\
\hline Bilirubin $(\mu \mathrm{mol} / \mathrm{l})$ & $19.4 \pm 11.2$ & $18.3 \pm 10.2$ & $20.6 \pm 10.8$ & 0.782 \\
\hline
\end{tabular}

HCC, hepatocellular carcinoma.
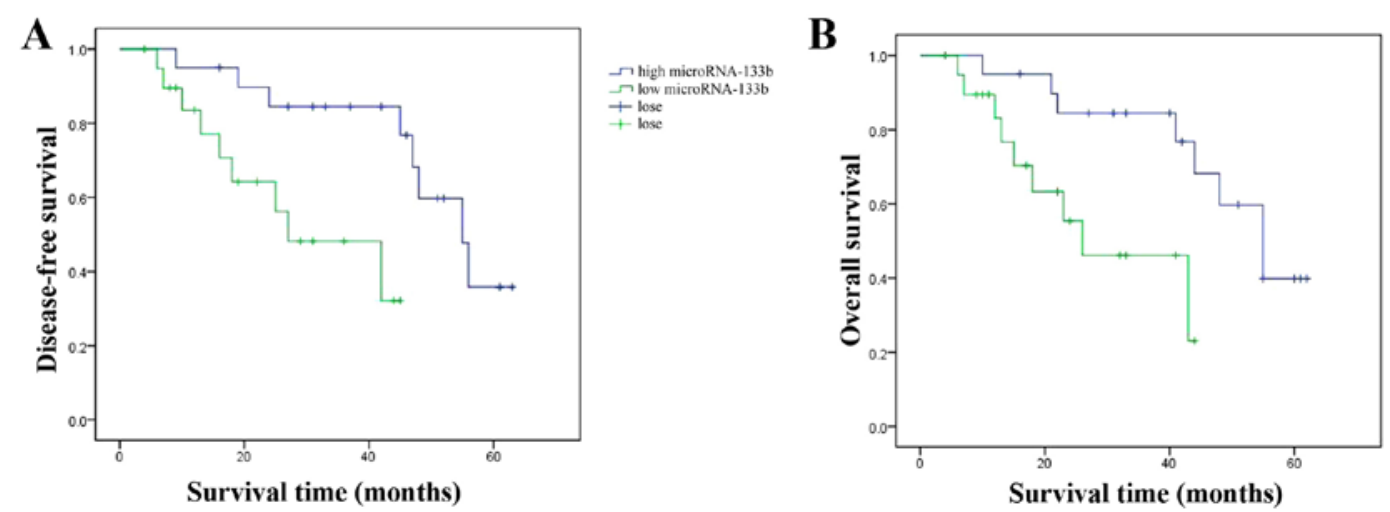

Figure 2. Effects of microRNA-133b expression on overall and disease-free survival of hepatocellular carcinoma patients. Effects of microRNA-133b expression on (A) disease-free and (B) overall survival of hepatocellular carcinoma patients.

patients with high miRNA-133b expression (Table II). The Edmondson grade and serum AFP levels of the HCC patients with low miRNA-133b expression were also higher than these parameters in the HCC patients with high miRNA-133b expression (Table II).

Effects of miRNA-133b expression on overall and disease-free survival of HCC patients. We aimed to ascertain the affects of miRNA-133b expression on the overall and disease-free survival of HCC patients. As shown in Fig. 2, the overall and disease-free survival of HCC patients with high miRNA-133b expression was observably extended, compared with the HCC patients with low miRNA-133b expression.

Expression of miRNA-133b in HCC cell lines. We detected the expression of miRNA-133b in HCC cell lines using RT-qPCR. As shown in Fig. 3, the expression of miRNA-133b in the
HepG2 cells was lowest among the HCC cell lines (HepG2, SMMC7721, Bel7404 and HCCM3). Thus, we selected the HepG2 cells for use in further experiments.

Overexpression of miRNA-133b inhibits the proliferation of and increases LDH activity in HepG2 cells. We investigated the effects of miRNA-133b on cell proliferation and LDH activity in HepG2 cells. As shown in Fig. 4, the overexpression of miRNA-133b significantly inhibited the proliferation of and increased LDH activity in the HepG2 cells, compared with these parameters in the negative group.

Overexpression of miRNA-133b induces the apoptosis of HepG2 cells. We confirmed the effects of miRNA-133b on the apoptosis of HepG2 cells. As shown in Fig. 5, the overexpression of miRNA-133b significantly induced the apoptosis of the HepG2 cells, compared with negative group. 


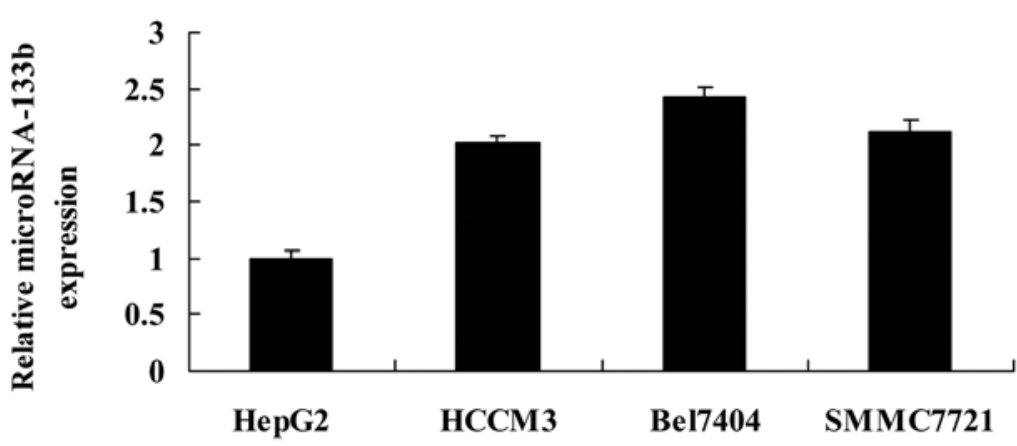

Figure 3. microRNA-133b expression in the HCC cell lines. HCC, hepatocellular carcinoma.
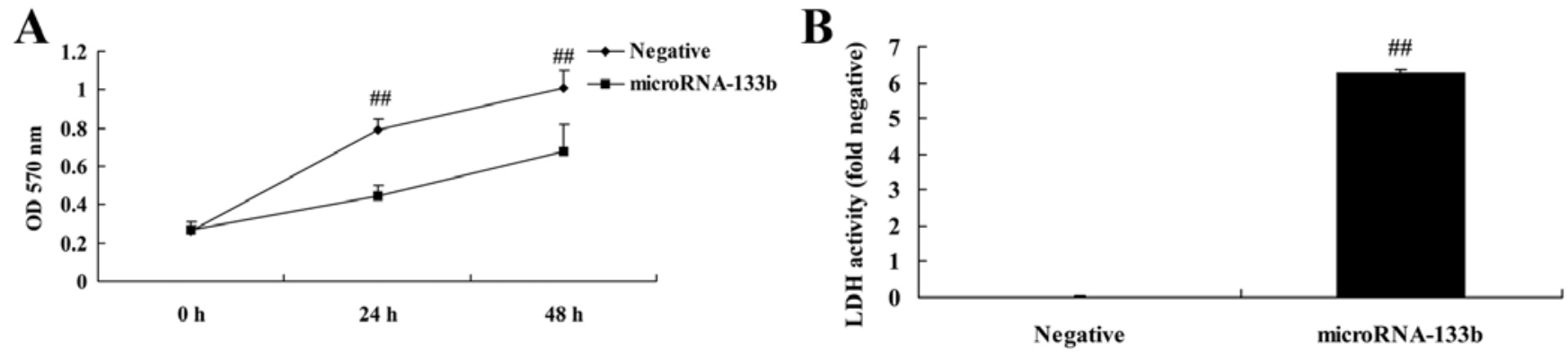

Figure 4. Overexpression of microRNA-133b inhibits cell proliferation and increases LDH activity in HepG2 cells. Overexpression of microRNA-133b (A) inhibited cell proliferation and (B) increased LDH activity in HepG2 cells; ${ }^{\# \#} \mathrm{p}<0.01$ compared with the negative group.

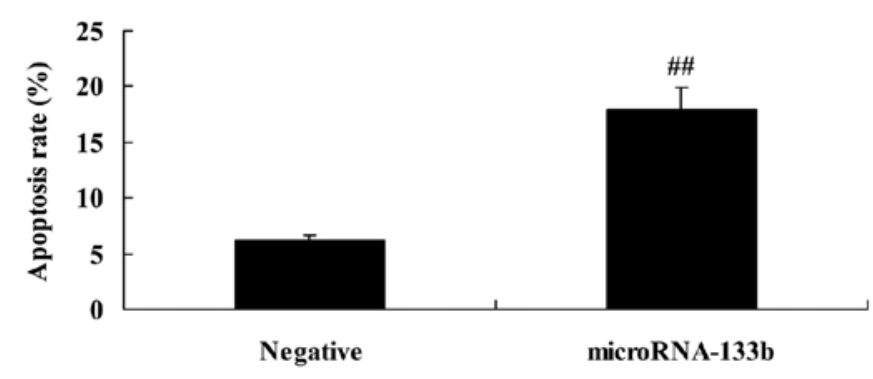

Figure 5. Overexpression of microRNA-133b induced the apoptosis of HepG2 cells; ${ }^{\# \#} \mathrm{p}<0.01$ compared with the negative group.

Overexpression of miRNA-133b promotes caspase-3/-8 activities and increases the Bax/Bcl-2 protein expression ratio in HepG2 cells. To explore the mechanisms underlying the induction of apoptosis in the HepG2 cells by miR-133b, we determined caspase- $3 /-8$ activities and Bax/Bcl-2 protein expression in the HepG2 cells. As shown in Fig. 6A-D, the overexpression of miRNA-133b significantly promoted caspase-3/-8 activities and increased the $\mathrm{Bax} / \mathrm{Bcl}-2$ protein expression ratio in the HepG2 cells, compared with the negative group.

Overexpression of miRNA-133b suppresses EGFR protein expression in HepG2 cells. To verify whether EGFR is a direct target of miRNA-133b, we measured EGFR protein expression by western blot analysis. As shown in Fig. 6C and E, the overexpression of miRNA-133b significantly suppressed EGFR protein expression in the HepG2 cells, compared with the negative group.

Overexpression of miRNA-133b suppresses PI3K, p-Akt and p-mTOR protein expression in HepG2 cells. Moreover, we verified that the PI3K/Akt/mTOR signaling pathway is a direct target of miRNA-133b. We assessed PI3K, Akt and mTOR protein expression by western blot analysis. As shown in Fig. 7, the overexpression of miRNA-133b significantly suppressed PI3K, p-Akt and p-mTOR protein expression in the HepG2 cells, compared with the negative group.

EGFR inhibitor enhances the suppressive effects on EGFR protein expression in HepG2 cells induced by the overexpression of miRNA-133b. To further investigate the role of EGFR in the effects of miRNA-133b on the poor of patients with HCC after curative hepatectomy, we downregulated EGFR expression using a EGFR inhibitor (GW2974, $2 \mu \mathrm{M})$. As shown in Fig. 8A and B, GW2974 significantly downregulated EGFR expression in the miRNA-133b-overexpressing HepG2 cells when compared with the miRNA-133b overexpression only group.

EGFR inhibitor enhances the inhibitory effects on cell proliferation induced by the overexpression of miRNA-133b and increases LDH activity even further in HepG2 cells. Furthermore, we investigated the effects of the downregulation of EGFR on the effects of miRNA-133b on the poor of 
A

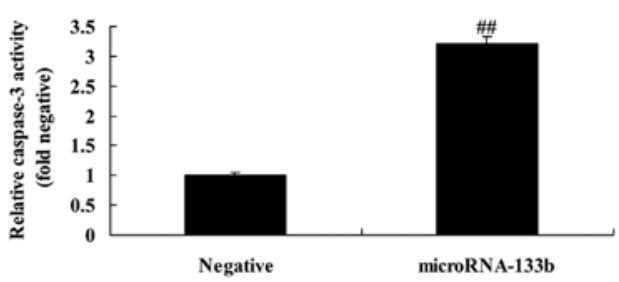

C

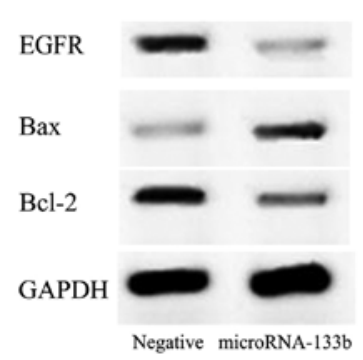

B

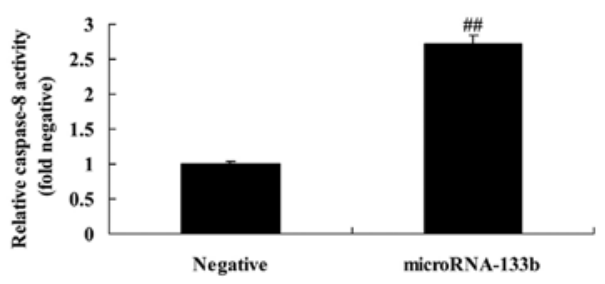

D

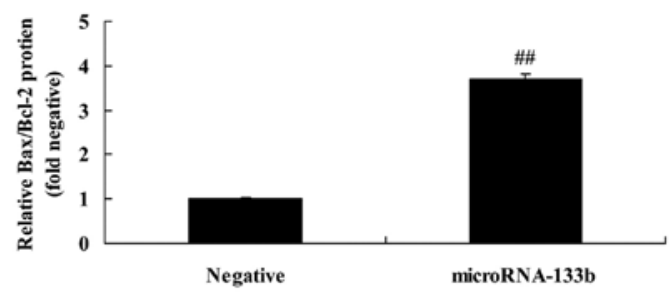

$\mathbf{E}$

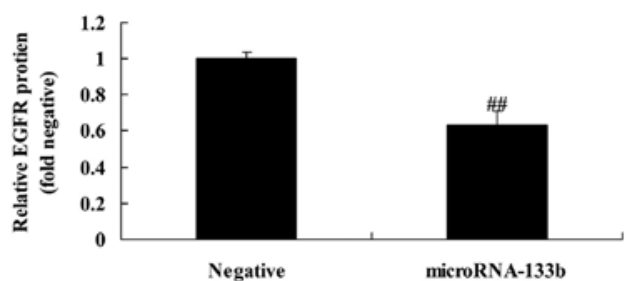

Figure 6. Overexpression of microRNA-133b promotes caspase-3/-8 activities, and increases Bax/Bcl-2 ratio and EGFR protein expression in HepG2 cells. Overexpression of microRNA-133b promoted (A and B) caspase-3/-8 activities and (C-E) increased the Bax/Bcl-2 ratio and EGFR protein expression as determined by western blot and statistical analyses of Bax/Bcl-2 and EGFR protein expression in HepG2 cells; ${ }^{* \#}$ p $<0.01$ compared with the negative group.

A

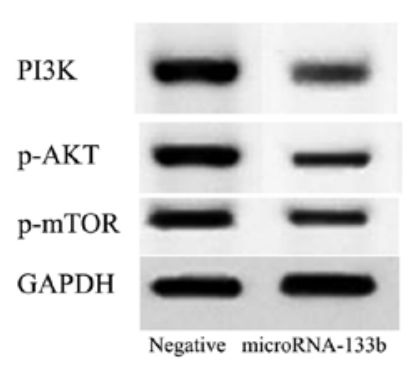

C

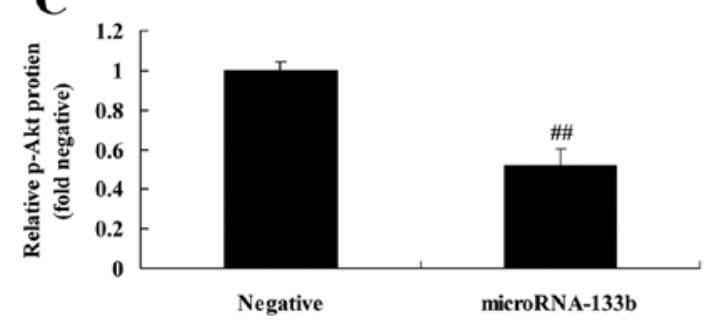

B

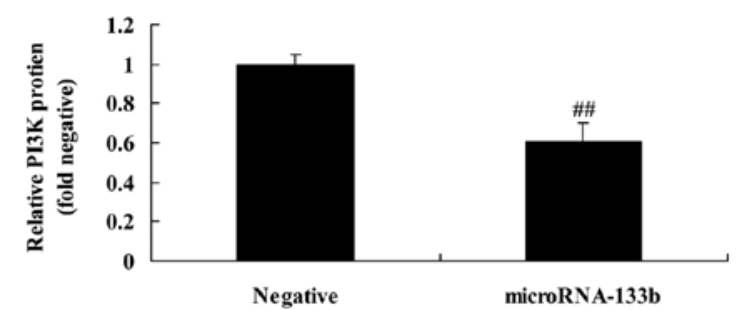

D

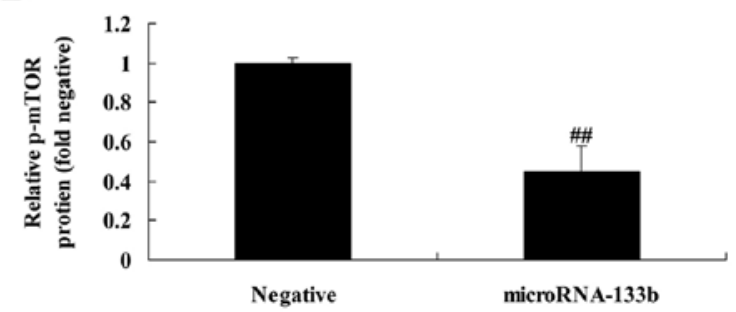

Figure 7. Overexpression of microRNA-133b suppresses PI3K, p-Akt and p-mTOR protein expression in HepG2 cells. Overexpression of microRNA-133b suppressed PI3K, p-Akt and p-mTOR protein expression as determined by (A) western blot and (B-D) statistical analyses, in HepG2 cells; ${ }^{\# \#}$ p $<0.01$ compared with the negative group.

patients with HCC after curative hepatectomy. As shown in Fig. 9, the downregulation of EGFR significantly inhibited cell proliferation and increased LDH activity in the miRNA-133b-overexpressing HepG2 cells compared with the miRNA-133b overexpression only group.
EGFR inhibitor enhances the promoting effects on the apoptosis of HepG2 cells induced by the overexpression of miRNA-133b. To further confirm whether the downregulation of EGFR mediates the promoting effects of miRNA-133b on the apoptosis of HepG2 cells, the apoptosis rate of HepG2 cells 
A

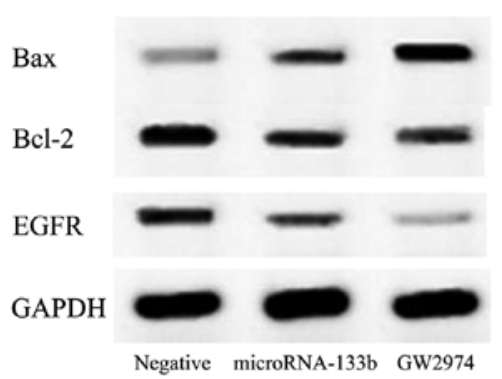

B

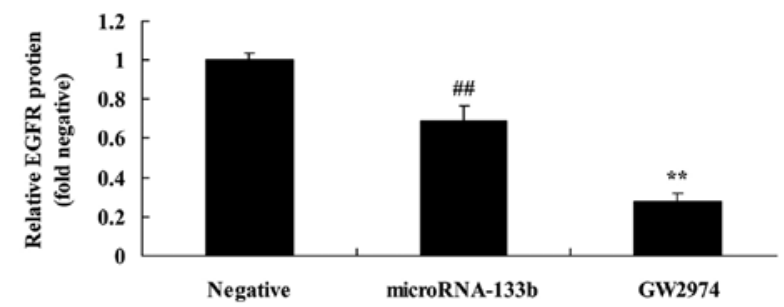

C

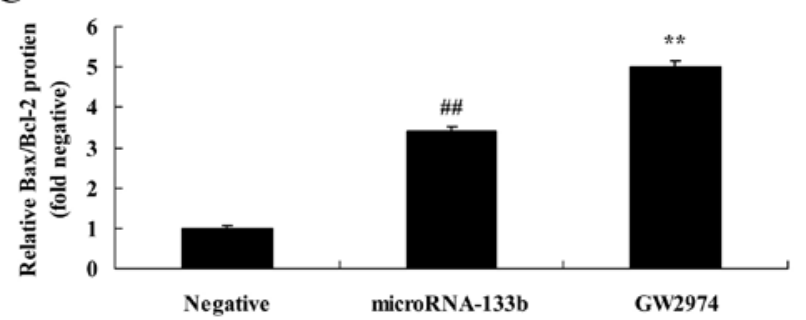

Figure 8. EGFR inhibitor suppresses EGFR and Bax/Bcl-2 protein expression ratio in HepG2 cells by overexpression of microRNA-133b. GFR inhibitor suppressed EGFR and Bax/Bcl-2 protein expression ratio using (A) western blot and (B and C) statistical analyses in HepG2 cells by overexpression of microRNA-133b; ${ }^{\# \#} \mathrm{p}<0.01$ compared with the negative group; ${ }^{* *} \mathrm{p}<0.01$ compared with the microRNA-133b overexpression only group.

A

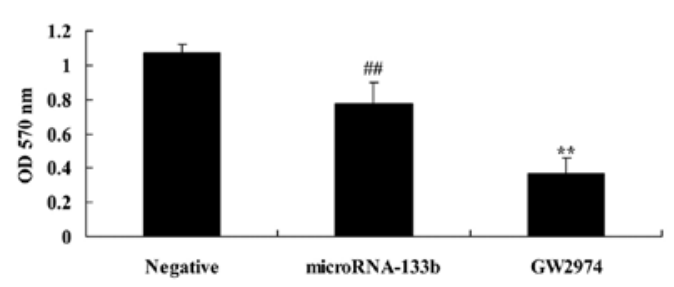

B

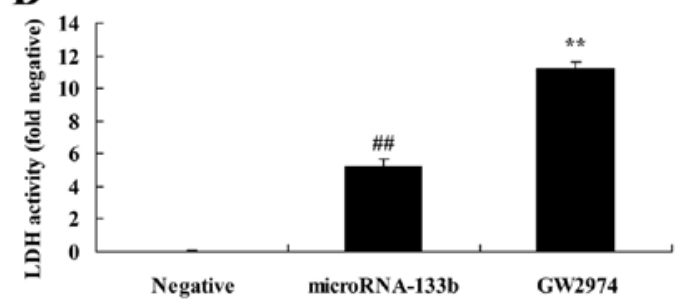

Figure 9. EGFR inhibitor inhibits cell proliferation and increases LDH activity in HepG2 cells by overexpression of microRNA-133b. EGFR inhibitor (A) inhibited cell proliferation and (B) increased LDH activity in HepG2 cells by overexpression of microRNA-133b; ${ }^{\# \#}$ p $<0.01$ compared with the negative group; ${ }^{* *} \mathrm{p}<0.01$ compared with the microRNA-133b overexpression only group.

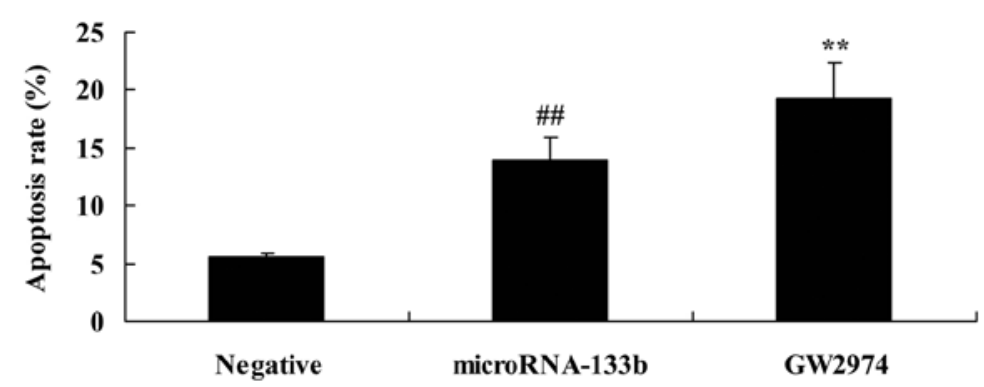

Figure 10. EGFR inhibitor induced the apoptosis of HepG2 cells by overexpression of microRNA-133b; ${ }^{\# \#} \mathrm{p}<0.01$ compared with the negative group; ${ }^{* *} \mathrm{p}<0.01$ compared with the microRNA-133b overexpression only group.

was measured by flow cytometry. As shown in Fig. 10, the downregulation of EGFR significantly induced the apoptosis of the miRNA-133b-overexpressing HepG2 cells, compared with the miRNA-133b overexpression only group.

EGFR inhibitor enhances the promoting effects on caspase-3/-8 activities and on the Bax/Bcl-2 protein expres- sion ratio in HepG2 cells induced by the overexpression of miRNA-133b. In HCC cells, when EGFR expression was downregulated, the effects on the apoptosis of HepG2 cells overexpressing miRNA-133b were assessed. As shown in Figs. 8A and $\mathrm{C}$, and 11, the downregulation of EGFR significantly promoted caspase-3/-8 activities and increased the $\mathrm{Bax} / \mathrm{Bcl}-2$ protein expression ratio in the miRNA-133b-over- 
A

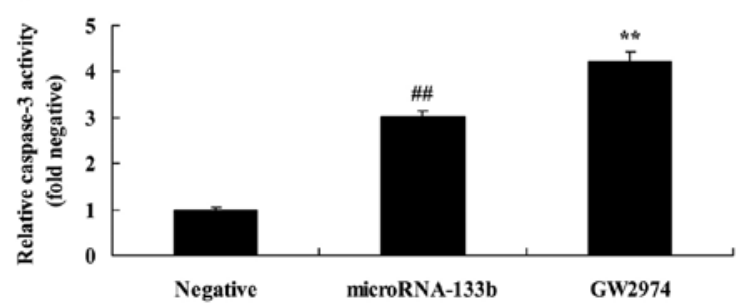

B

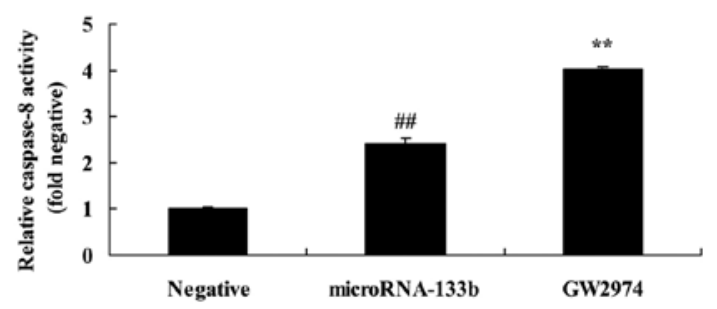

Figure 11. EGFR inhibitor promotes caspase-3/-8 activities in HepG2 cells by overexpression of microRNA-133b. (A and B) EGFR inhibitor promoted (A) caspase-3 and (B) caspase- 8 activities in HepG2 cells by overexpression of microRNA-133b; ${ }^{\# \#}$ p $<0.01$ compared with the negative group; ${ }^{* *}$ p $<0.01$ compared with the microRNA-133b overexpression only group.

A

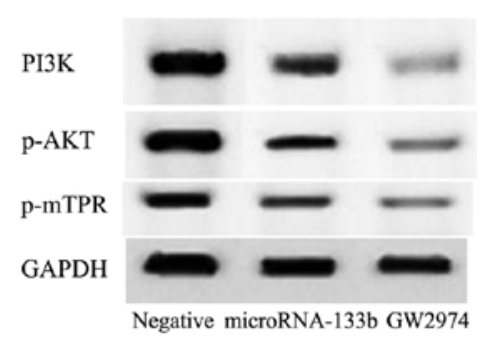

C

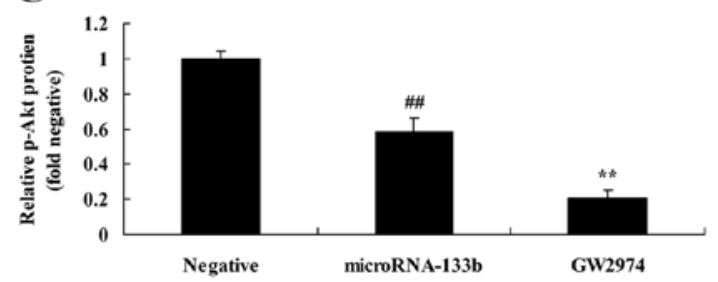

B

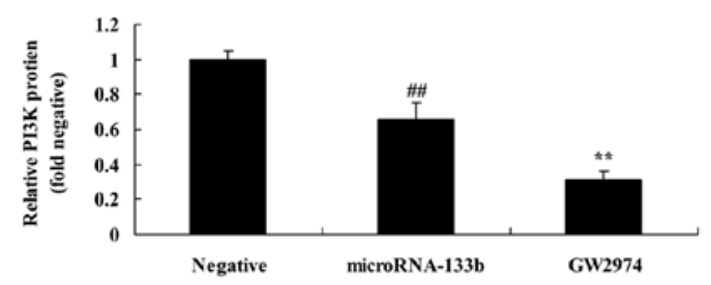

D

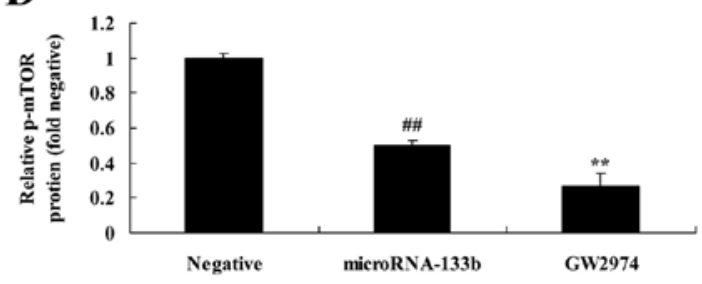

Figure 12. EGFR inhibitor suppresses PI3K, p-Akt and p-mTOR protein expression in HepG2 cell by overexpression of microRNA-133b. EGFR inhibitor suppresses PI3K, p-Akt and p-mTOR protein expression using (A) western blot and (B-D) statistical analyses in HepG2 cells by overexpression of microRNA$133 \mathrm{~b} ;{ }^{\# \#} \mathrm{p}<0.01$ compared with the negative group; ${ }^{* *} \mathrm{p}<0.01$ compared with the microRNA-133b overexpression only group.

expressing HepG2 cells compared with the miRNA-133b overexpression only group.

EGFR inhibitor enhances the suppressive effects on PI3K, $p$-Akt and p-mTOR protein expression in Hep 2 cells induced by the overexpression of miRNA-133b. To examine whether EGFR regulates miRNA-133b in regards to HepG2 cell growth, we suppressed EGFR expression and then analyzed the effects on the PI3K/Akt/mTOR signaling pathway. As shown in Fig. 12, the downregulation of EGFR significantly downregulated the PI3K/Akt/mTOR signaling pathway in miRNA-133b-overexpressing HepG2 cells compared with the miRNA-133b overexpression only group.

\section{Discussion}

Hepatocellular carcinoma is one the most common malignant tumors. Its incidence is increasing. Due to the technological development of hepatic surgery and the establishment of new treatments, the disease-free survival of patients has been extended (24). However, the recurrence rate remains high. Various foreign documents report that the recurrence rate of $\mathrm{HCC}$ can reach $70-80 \%$ within 5 years after partial hepatectomy. According to domestic documents, the recurrence rate can be up to $57-81 \%$ within 3 years after hepatectomy. The results of the present study showed that the overexpression of miRNA-133b inhibited cell proliferation, increased LDH activity, induced apoptosis and promoted caspase-3/-8 activities and Bax/Bcl-2 protein expression in HepG2 cells.

The activation or mutation of EGFR may cause the cascade of signaling pathways downstream and finally induce uncontrollable proliferation of tumor cells (19). The overexpression of EGFR is likely to be a marker of independent prognosis related to the proliferation of tumor cells, reduction of radiosensitivity and high recurrence rate of tumors (25). EGFR gene amplification induces overexpression of EGFR (25). EGFR is able to cause excessive activation of its kinase through spontaneous dimerization (18). In the present study, the overexpression of miRNA-133b significantly suppressed EGFR protein expression in HepG2 cells.

The excessive activation of PI3K plays a critical role in the occurrence and development of HCC (26). There are a number of different mechanisms mediating the upregulation of PI3K expression. LMP1 is able to activate PI3K directly, thereby causing the phosphorylation of Akt (26). With the activation of NF- $\kappa \mathrm{B}$, the TRAF binding domain in LMP1 becomes 


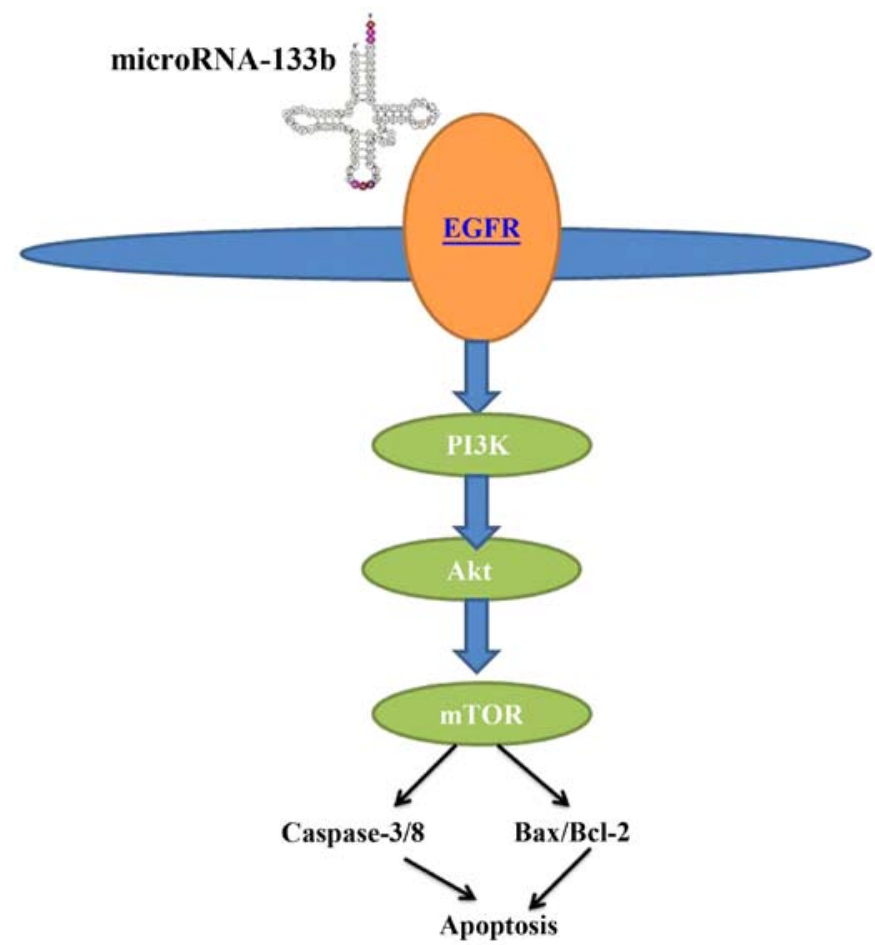

Figure 13. Overexpression of microRNA-133b increases the survival of patients with hepatocellular carcinoma after curative hepatectomy through the EGFR/ $\mathrm{PI} 3 \mathrm{~K} / \mathrm{Akt} / \mathrm{mTOR}$ signaling pathway.

the active site (20). The activation of the phosphorylation of Akt can further induce the phosphorylation of downstream molecules (27). In addition, it may make them participate in cellular metabolism, proliferation, survival and growth. Upstream molecules of Atk are blocked by PDK1, which effectively inhibit the growth of tumor cells. Most HCC cases have sustained activation (26). The phosphorylation and overexpression of Akt can be detected in HCC tissues and HCC cell lines.

Activated mTOR can regulate key factors of protein translation such as p70S6K and 4EBP1 (28). The latter relieves the depression of eIF4E and finally induces the translation of a series of proteins that promote cell growth. EIF4E gene amplification and overexpression are closely linked with the clinical progression of HCC (29). EMT is obviously inhibited in HCC cells treated with PI3K and mTOR inhibitors (30). Thus, EMT of tumor cells is possibly connected with the PI3K/mTOR pathways (30). In the present study, we found that the overexpression of miRNA-133b significantly suppressed PI3K, p-Akt and p-mTOR protein expression in HepG2 cells.

The EGFR/PI3K/Akt/mTOR signaling pathway plays an important role in the occurrence and development of HCC (31). Key factors of this signaling pathway have become a research focus. Related drugs are undergoing pre-clinical trials or clinical trials (32). However, due to the complexity of this signaling pathway and interaction among different signaling pathways, the molecular-targeted therapy for HCC merely serves as an alternative therapy for conventional radiotherapy and chemotherapy or adjuvant treatment based on conventional radiotherapy and chemotherapy $(33,34)$. Our results showed that the suppression of EGFR inhibited cell proliferation, increased LDH activity, induced apoptosis and promoted caspase-3/-8 activities and increased Bax/Bcl-2 protein expression ratio, downregulated PI3K, phosphorylated p-Akt and phosphorylated-p-mTOR protein expression in the transfected HCC cells overexpressing miRNA-133b.

In conclusion, our results suggest that the overexpression of miRNA-133b increases the survival of patients with HCC after curative hepatectomy, and thus plays protective a role in HCC progression. miRNA-133b may thus serve as a novel prognostic biomarker in patients with $\mathrm{HCC}$, namely that patients with a low expression of this miRNA are predicted to have a poorer survival. Our findings also indicated that the promoting effects of miRNA-133b on the survival of patients with HCC, as well as its suppressive effects on the survival of HCC HepG2 cells are mediated through the EGFR/PI3K/Akt/ mTOR signaling pathway (Fig. 13). These findings provide a potential therapeutic target for HCC treatment.

\section{Acknowledgements}

The present study was supported by the First-Class General Financial Grant from the China Postdoctoral Science Foundation (grant no. 2014M562551).

\section{References}

1. Geissler EK, Schnitzbauer AA, Zülke C, Lamby PE, Proneth A, Duvoux C, Burra P, Jauch KW, Rentsch M, Ganten TM, et al: Sirolimus use in liver transplant recipients with hepatocellular carcinoma: A randomized, multicenter, open-label phase 3 trial. Transplantation 100: 116-125, 2016.

2. Erhardt A, Kolligs F, Dollinger M, Schott E, Wege H, Bitzer M, Gog C, Lammert F, Schuchmann M, Walter C, et al: TACE plus sorafenib for the treatment of hepatocellular carcinoma: Results of the multicenter, phase II SOCRATES trial. Cancer Chemother Pharmacol 74: 947-954, 2014.

3. Zhang L, Ge NL, Chen Y, Xie XY, Yin X, Gan YH, Zhang BH, Zhang JB, Chen RX, Wang YH, et al: Long-term outcomes and prognostic analysis of radiofrequency ablation for small hepatocellular carcinoma: 10-year follow-up in Chinese patients. Med Oncol 32: 77, 2015.

4. Hirokawa F, Hayashi M, Miyamoto Y, Asakuma M, Shimizu T, Komeda K, Inoue Y and Uchiyama K: Predictors of poor prognosis by recurrence patterns after curative hepatectomy for hepatocellular carcinoma in Child-Pugh classification A. Hepatogastroenterology 62: 164-168, 2015.

5. Hu BS, Zhao G, Yu HF, Chen K, Dong JH and Tan JW: High expression of AP-4 predicts poor prognosis for hepatocellular carcinoma after curative hepatectomy. Tumour Biol 34: 271-276, 2013.

6. Liu F, Zhang Y, Peng Z, Gao H, Xu L and Chen M: High expression of high mobility group box 1 (hmgbl) predicts poor prognosis for hepatocellular carcinoma after curative hepatectomy. J Transl Med 10: 135, 2012.

7. Wang C, Xiang H, Si H, Guo D and Sun M: High expression of myofibrillogenesis regulator-1 predicts poor prognosis for patients with hepatocellular carcinoma after curative hepatectomy. Int J Clin Exp Pathol 8: 14818-14823, 2015.

8. Hirokawa F, Hayashi M, Asakuma M, Shimizu T, Inoue Y and Uchiyama K: Risk factors and patterns of early recurrence after curative hepatectomy for hepatocellular carcinoma. Surg Oncol 25: 24-29, 2016.

9. Yang SL, Liu LP, Sun YF, Yang XR, Fan J, Ren JW, Chen GG and Lai PB: Distinguished prognosis after hepatectomy of HBV-related hepatocellular carcinoma with or without cirrhosis: A long-term follow-up analysis. J Gastroenterol 51: 722-732, 2016.

10. Wada H, Yamamoto H, Kim C, Uemura M, Akita H, Tomimaru Y, Hama N, Kawamoto K, Kobayashi S, Eguchi H, et al: Association between ephrin-A1 mRNA expression and poor prognosis after hepatectomy to treat hepatocellular carcinoma. Int J Oncol 45: 1051-1058, 2014. 
11. Kobayashi A, Takahashi S, Ishii H, Konishi M, Nakagohri T, Gotohda N,Satake M,Furuse J and Kinoshita T: Factors predicting survival in advanced T-staged hepatocellular carcinoma patients treated with reduction hepatectomy followed by transcatheter arterial chemoembolization. Eur J Surg Oncol 33: 1019-1024, 2007.

12. Ahn S, Hyeon $\mathbf{J}$ and Park CK: Metadherin is a prognostic predictor of hepatocellular carcinoma after curative hepatectomy. Gut Liver 7: 206-212, 2013.

13. Mizuguchi Y, Takizawa T, Yoshida H and Uchida E: Dysregulated miRNA in progression of hepatocellular carcinoma: A systematic review. Hepatol Res 46: 391-406, 2016.

14. Huang JT, Liu SM, Ma H, Yang Y, Zhang X, Sun H, Zhang X, $\mathrm{Xu} \mathrm{J}$ and Wang J: Systematic review and meta-analysis: Circulating miRNAs for diagnosis of hepatocellular carcinoma. J Cell Physiol 231: 328-335, 2016.

15. Mahgoub A and Steer CJ: MicroRNAs in the evaluation and potential treatment of liver diseases. J Clin Med 5: pii: E52, 2016.

16. Leng C, Zhang ZG, Chen WX, Luo HP, Song J, Dong W, Zhu XR, Chen XP, Liang HF and Zhang BX: An integrin beta4-EGFR unit promotes hepatocellular carcinoma lung metastases by enhancing anchorage independence through activation of FAK-AKT pathway. Cancer Lett 376: 188-196, 2016.

17. Wang YP, Huang LY, Sun WM, Zhang ZZ, Fang JZ, Wei BF, Wu BH and Han ZG: Insulin receptor tyrosine kinase substrate activates EGFR/ERK signalling pathway and promotes cell proliferation of hepatocellular carcinoma. Cancer Lett 337: 96-106, 2013

18. Hu H, Gao L, Wang C, Li Y, Ma H, Chen L, Qin J, Liu B, Liu Y and Liang C: Lower serum soluble-EGFR is a potential biomarker for metastasis of HCC demonstrated by $\mathrm{N}$-glycoproteomic analysis. Discov Med 19: 333-341, 2015.

19. Li T, Dong ZR, Guo ZY, Wang CH, Zhi XT, Zhou JW, Li DK, Chen ZT, Chen ZQ and Hu SY: Mannose-mediated inhibitory effects of PA-MSHA on invasion and metastasis of hepatocellular carcinoma via EGFR/Akt/I $\kappa \mathrm{B} \beta / \mathrm{NF}-\kappa \mathrm{B}$ pathway. Liver Int 35: 1416-1429, 2015.

20. Jiang J, Zhang Y, Guo Y, Yu C, Chen M, Li Z, Tian S and Sun C: MicroRNA-3127 promotes cell proliferation and tumorigenicity in hepatocellular carcinoma by disrupting of PI3K/AKT negative regulation. Oncotarget 6: 6359-6372, 2015.

21. Pellegrino R, Calvisi DF, Neumann O, Kolluru V, Wesely J, Chen X, Wang C, Wuestefeld T, Ladu S, Elgohary N, et al: EEF1A2 inactivates p53 by way of PI3K/AKT/mTOR-dependent stabilization of MDM4 in hepatocellular carcinoma. Hepatology 59: 1886-1899, 2014.

22. Zhu M, Guo J, Li W, Xia H, Lu Y, Dong X, Chen Y, Xie X, Fu S and $\mathrm{Li} \mathrm{M}$ : HBx induced AFP receptor expressed to activate $\mathrm{PI} 3 \mathrm{~K} / \mathrm{AKT}$ signal to promote expression of Src in liver cells and hepatoma cells. BMC Cancer 15: 362, 2015.

23. Huang Q, Zhan L, Cao H, Li J, Lyu Y, Guo X, Zhang J, Ji L, Ren T, An J, et al: Increased mitochondrial fission promotes autophagy and hepatocellular carcinoma cell survival through the ROS-modulated coordinated regulation of the NFKB and TP53 pathways. Autophagy 12: 999-1014, 2016.
24. Brandi G, de Rosa F, Agostini V, di Girolamo S, Andreone P, Bolondi L, Serra C, Sama C, Golfieri R, Gramenzi A, et al; Italian Liver Cancer (ITA.LI.CA) Group: Metronomic capecitabine in advanced hepatocellular carcinoma patients: A phase II study. Oncologist 18: 1256-1257, 2013.

25. Lanaya H, Natarajan A, Komposch K, Li L, Amberg N, Chen L, Wculek SK, Hammer M, Zenz R, Peck-Radosavljevic M, et al: EGFR has a tumour-promoting role in liver macrophages during hepatocellular carcinoma formation. Nat Cell Biol 16: 972-981, 2014.

26. Xu J, Jia L, Ma H, Li Y, Ma Z and Zhao Y: Axl gene knockdown inhibits the metastasis properties of hepatocellular carcinoma via PI3K/Akt-PAK1 signal pathway. Tumour Biol 35: 3809-3817, 2014.

27. Jiang X, Zeng L, Huang J, Zhou H and Liu Y: Arctigenin, a natural lignan compound, induces apoptotic death of hepatocellular carcinoma cells via suppression of PI3-K/Akt signaling. J Biochem Mol Toxicol 29: 458-464, 2015.

28. Wang $\mathrm{H}$, Zhang $\mathrm{C}$, Xu L, Zang K, Ning Z, Jiang F, Chi H, Zhu X and Meng Z: Bufalin suppresses hepatocellular carcinoma invasion and metastasis by targeting HIF-1 $\alpha$ via the PI3K/AKT/mTOR pathway. Oncotarget 7: 20193-20208, 2016.

29. Nemazanyy I, Espeillac C, Pende M and Panasyuk G: Role of PI3K, mTOR and Akt2 signalling in hepatic tumorigenesis via the control of PKM2 expression. Biochem Soc Trans 41: 917-922, 2013.

30. Zhang Y, Guo X, Xiong L, Yu L, Li Z, Guo Q, Li Z, Li B and Lin N: Comprehensive analysis of microRNA-regulated protein interaction network reveals the tumor suppressive role of microRNA-149 in human hepatocellular carcinoma via targeting AKT-mTOR pathway. Mol Cancer 13: 253, 2014.

31. Horn D, Hess J, Freier K, Hoffmann J and Freudlsperger C: Targeting EGFR-PI3K-AKT-mTOR signaling enhances radiosensitivity in head and neck squamous cell carcinoma. Expert Opin Ther Targets 19: 795-805, 2015.

32. Makinoshima $H$, Takita $M$, Saruwatari K, Umemura $S$, Obata Y, Ishii G, Matsumoto S, Sugiyama E, Ochiai A, Abe R, et al: Signaling through the phosphatidylinositol 3-kinase $(\mathrm{PI} 3 \mathrm{~K}) / \mathrm{mammalian}$ target of rapamycin (mTOR) axis is responsible for aerobic glycolysis mediated by glucose transporter in epidermal growth factor receptor (EGFR)-mutated lung adenocarcinoma. J Biol Chem 290: 17495-17504, 2015.

33. Ooft ML, Braunius WW, Heus P, Stegeman I, van Diest PJ, Grolman W, Zuur CI and Mayems SM: Prognostic significance of the EGFR pathway in nasopharyngeal carcinoma: A systematic review and meta-analysis. Biomarkers Med 9: 997-1010, 2015.

34. Brouxhon SM, Kyrkanides S, Teng X, Athar M, Ghazizadeh S, Simon M, O'Banion MK and Ma L: Soluble E-cadherin: A critical oncogene modulating receptor tyrosine kinases, MAPK and PI3K/Akt/mTOR signaling. Oncogene 33: 225-235, 2014. 